\title{
Identification of a microRNA signature in endothelial cells with mechanical stretch stimulation
}

\author{
JUBING ZHENG $^{1}$, KUI ZHANG ${ }^{1}$, YUELI WANG ${ }^{2}$, JIAN CAO ${ }^{1}$, \\ FENG ZHANG $^{3}$, QIWEN ZHOU ${ }^{1}$ and RAN DONG ${ }^{1}$
}

\begin{abstract}
Departments of ${ }^{1}$ Cardiac Surgery and ${ }^{2}$ Cardiology, Beijing Institute of Heart, Lung and Blood Vessel Disease, Capital Medical University, Beijing Anzhen Hospital, Beijing 100029; ${ }^{3}$ Department of Bioinfomatics, Beijing Institute of Genomics, Chinese Academy of Sciences, Beijing 100101, P.R. China
\end{abstract}

Received May 20, 2014; Accepted February 5, 2015

DOI: $10.3892 / \mathrm{mmr} .2015 .3835$

\begin{abstract}
The current study aimed to verify an miRNA signature in endothelial cells undergoing mechanical stretch stimulation. In the present study, microarray profiling was conducted in order to identify the differential expression of miRNAs in endothelial cells undergoing mechanical stimulation, compared with unstimulated endothelial cells. The microarray data was then validated by reverse transcription-quantitative polymerase chain reaction. Genes and signaling pathways regulated by the miRNAs were investigated in silico using Gene Ontology and the Kyoto Encyclopedia of Genes or Genomes, which are ontological and network-mapping algorithms. The microarray data collected demonstrated that 38 miRNAs exhibited significant differential expression in endothelial cells with mechanical stretch stimulation. Of these, 20 were upregulated and 18 were downregulated. The results from the in silico analysis indicated that the miRNAs identified were participants in mechanical stretch-induced endothelial dysfunction. During the initial stage of vein graft failure, which is induced by endothelial dysfunction, a unique miRNA signature was identified. The identified miRNAs are suggested to be involved in the pathological processes of traumatic injury.
\end{abstract}

Correspondence to: Dr Ran Dong or Dr Qiwen Zhou, Department of Cardiac Surgery, Beijing Institute of Heart, Lung and Blood Vessel Disease, Capital Medical University, Beijing Anzhen Hospital, 2 Anzhen Road, Beijing 100029, P.R. China

E-mail: rdong_az@126.com

E-mail: qiwen_zhou@163.com

Abbreviations: CABG, coronary artery bypass graft; VGF, vein graft failure; EC, endothelial cell; miRNAs, microRNAs; EGR1, early growth response 1; RT-qPCR, reverse transcription-quantitative polymerase chain reaction; GO, Gene Ontology; KEGG, Kyoto Encyclopedia of Genes and Genomes; VSMC, vascular smooth muscle cell; MAPK, mitogen-activated protein kinase

Key words: microRNA signature, endothelial cells, mechanical stretch stimulation, microarray profiling

\section{Introduction}

Compared with percutaneous coronary intervention and medical therapy, coronary artery bypass graft (CABG) has been demonstrated to be a more effective method for the treatment of coronary artery disease (1). The saphenous vein is the most commonly used vessel for the graft in CABG surgery; however, vein graft failure (VGF) is a serious complication that affects the curative efficacy of CABG. Endothelial dysfunction is a critical factor in VGF (2). Following CABG, the vein graft wall sustains high blood pressure and is frequently injured by mechanical stretch. This injury leads to endothelial dysfunction, which in turn induces alterations in endothelial cell (EC) proliferation, cytokine secretion, leukocyte adherence and platelet aggregation (3-5). However, the mechanism underlying endothelial dysfunction remains unclear.

MicroRNAs (miRNAs) are small non-coding RNA molecules, 22 nucleotides in length, that are crucial in cell growth, differentiation, metabolism, apoptosis and migration (6). In a previous study, it was demonstrated that early growth response 1 (EGR1) is a vital factor in the development of VGF. Additionally, certain miRNAs that were able to regulate EGR1 expression were predicted via miRBase (http://mirbase.org), Target Scan Human (http://www.targetscan.org/) and miRDB (http://mirdb.org/miRDB/) (Zheng et al, unpublished data). Several previous studies have suggested that miRNAs are involved in various pathological cardiovascular conditions, including atherosclerosis, hypertension and arrhythmia (7-9). In 2013, McDonald et al (10) demonstrated that in VGF, miR-21 induces intimal hyperplasia by regulating the expression of the phosphatase and tensin homolog deleted on chromosome 10, signal transducer and activator of transcription 3 and proliferating cell nuclear antigen. These results suggested that miRNAs are vital regulated factors and may be used as novel genetic therapeutic targets in the treatment of VGF (11). However, in endothelial dysfunction induced by mechanical stretch, the expression and mechanistic regulation of EC miRNAs remains unclear. An improved understanding of miRNA expression in ECs with mechanical stretch stimulation may lead to the development of novel biomarkers and therapeutic targets for the initial stages of VGF. Therefore, in order to identify miRNA expression in endothelial dysfunction, 
the current study conducted miRNA profiling using samples obtained from ECs undergoing mechanical stretch.

\section{Materials and methods}

Mechanical stretch stimulation. Human umbilical vein endothelial cells (HUVECs; GeneChem Co., Ltd., Shanghai, China) were maintained in Dulbecco's modified Eagle's medium 140 (Gibco Life Technologies, Grand Island, NY, USA) with $10 \%$ fetal bovine serum (Gibco Life Technologies) at $37^{\circ} \mathrm{C}$ with $5 \% \mathrm{CO}_{2}$. The ECs were then plated on silicone elastomer-bottomed and collagen-coated plates (Flexcell International Corporation, McKeesport, PA, USA). A computer-controlled Cyclic Stress Unit (Flexcell 5100; Flexcell International Corporation) was used to subject HUVECs to mechanical stretch for $24 \mathrm{~h}$ (cyclic deformation at 60 cycles/min, elongation of $15 \%$ ).

RNA isolation. Total RNA was isolated with the mirVana miRNA Isolation kit (Applied Biosystems, Foster City, CA, USA) in accordance with the manufacturer's instructions. RNA quantity and purity was assessed using a NanoDrop ND-1000 spectrophotometer (Thermo Fisher Scientific, Wilmington, MA, USA). A260/A280 $\geq 1.6$ and A260/A230 $\geq 1$ indicated acceptable RNA purity, while an acceptable RNA integrity number $\geq 5$ was obtained using the Agilent RNA 6000 Nano assay (Agilent Technologies, Inc., Santa Clara, CA, USA). gDNA contamination was evaluated by gel electrophoresis.

Microarray profiling. Human genome-wide miRNA microarray analysis was performed by Phalanx Biotech with the Human miRNA OneArray ${ }^{\circledR}$ V4 (Phalanx Biotech Group, Hsinchu, Taiwan, R.O.C.). Each microarray contained 144 experimental control probes and 1,884 unique human miRNA probes. Each unique probe had three features, and probes contained 100\% Sanger miRBase V18 miRNA content. Briefly, fluorescent targets were prepared from $2.5 \mu \mathrm{g}$ total RNA samples using the miRNA ULSTM Labeling kit (Leica Biosystems Nussloch GmbH, Nussloch, Germany). Labeled miRNA targets were hybridized to the microarray with Phalanx hybridization buffer in the OneArray ${ }^{\circledR}$ Hybridization Chamber. Following overnight hybridization at $37^{\circ} \mathrm{C}$, non-specific binding was removed by three washing steps (wash one, $37^{\circ} \mathrm{C}, 5 \mathrm{~min}$; wash two, $37^{\circ} \mathrm{C}, 5 \mathrm{~min}$ and $25^{\circ} \mathrm{C}, 5 \mathrm{~min}$; and wash three: rinse 20 times) with $2 \mathrm{X}$ saline sodium citrate solution (Loogene, Beijing, China). The slides were dried by centrifugation at $70 \mathrm{x} \mathrm{g}$ for $5 \mathrm{~min}$ and scanned using an Axon GenePix ${ }^{\circledR} 4000 B$ scanner (Molecular Devices, Sunnyvale, CA, USA). The Cy5 fluorescence intensity of each spot was analyzed using GenePix, version 4.1 software (Molecular Devices). The signal intensity of each spot was processed using the R project, version 2.12.1 (Bell Laboratories, Murray Hill, NJ, USA). Spots for which the flag was $<0$ were filtered out, whereas spots that passed the criteria were normalized using the $75 \%$ scaling normalization method. Normalized spot intensities were then converted into $\log 2$ ratios of gene expression between the control and treatment groups. Spots with a $\log 2$ ratio of $\geq 1$ or $\leq-1$, in addition to a $\mathrm{P}<0.05$ were analyzed further. These differentially expressed miRNAs were subjected to hierarchical cluster analysis using average linkage and Pearson's correlation in order to measure the similarity.

Validation of microRNAs by reverse transcription-quantitative polymerase chain reaction $(R T-q P C R)$ analysis. The differentially expressed miRNAs selected from the microarray were validated by RT-qPCR analysis. cDNA was generated with the PrimeScript ${ }^{\mathrm{TM}}$ RT-qPCR kit (Takara Bio, Inc., Otsu, Japan) in accordance with the manufacturer's instructions. PCR was performed in a 7900HT fast RT-qPCR instrument (Applied Biosystems Life Technologies, Singapore). The primers used were purchased from Ribobio (Guangzhou, China) and the sequences for the control were as follows: U6, forward 5'-GCGCGTCGTGAAGCGTTC -3 ' and reverse 5'-GTGCAGGGTCCGAGGT-3'.All reactions involved initial denaturation at $95^{\circ} \mathrm{C}$ for $3 \mathrm{~min}$, followed by 40 cycles of $95^{\circ} \mathrm{C}$ for $10 \mathrm{sec}, 60^{\circ} \mathrm{C}$ for $20 \mathrm{sec}$ and $70^{\circ} \mathrm{C}$ for 1 sec. miRNA quantification was performed by using the miDETECT A TrackTM miRNA qRT-PCR Starter Kit (Ribobio) in a 7900HT fast RT-qPCR instrument (Applied Biosystems, Foster City, CA, USA). All quantitative reactions, including the no-template controls, were run in duplicate. The small nuclear RNA U6 was used as the normalization control.

Gene Ontology (GO) and Kyoto Encyclopedia of Genes and Genomes (KEGG) pathway analyses. In order to identify possible mRNA targets of the differentially expressed miRNAs, GO and KEGG were performed using miRBase (http://www.mirbase.org/). Enrichment analysis of miRNA target genes was performed using Cytoscape (http://www. cytoscape.org/). Using $\mathrm{P}<0.05$ as a cutoff, three different annotations were analyzed: Molecular function, biological process and cellular component.

The DAVID database (http://david.abcc.ncifcrf.gov/) was utilized in order to analyze the significant pathways that involved genes that were differentially expressed in HUVECs undergoing stimulation by mechanical stretch. Selection was based on the results of Fisher's exact test and the threshold of significance was defined by the P-value.

Statistical analysis. Data are expressed as the mean \pm standard error. Student's t-test was used to determine the significance of differences in miRNA expression between groups. All statistical analyses were performed using SPSS, version 17.0 (SPSS, Inc., Chicago, IL, USA). $\mathrm{P}<0.05$ was considered to indicate a statistically significant difference. $\mathrm{R}$ project was used to normalize data for the selection of differentially expressed miRNAs.

\section{Results}

miRNA signature in HUVECs following mechanical stretch stimulation. A total of 5,796 probes were included in the miRNA microarray that was used in the current study. miRNA microarray hybridization revealed that a total of 38 miRNAs were significantly differentially expressed following mechanical stretch stimulation. Of these, 20 were upregulated and 18 were downregulated (based on a fold change $\geq 0.8$ and $\mathrm{P}<0.05$; Table I). The differential miRNA expression patterns of HUVECs stimulated by mechanical stretch were accurately 
Table I. Differentially expressed miRNAs in human umbilical vein endothelial cells undergoing mechanical stretch stimulation, selected at $\log 2 \mid$ Fold changel $\geq 0.8$ and $\mathrm{P}<0.05$.

\begin{tabular}{lcc}
\hline Name & Fold change & P-value \\
\hline hsa-miR-363-5p & -1.3723 & 0.0148 \\
hsa-miR-668 & -1.3153 & 0.0030 \\
hsa-miR-4638-5p & -1.2859 & 0.0196 \\
hsa-miR-16-5p & -1.1988 & 0.0177 \\
hsa-miR-3676-3p & -1.1492 & 0.0029 \\
hsa-miR-3175 & -1.0751 & 0.0007 \\
hsa-miR-106a-5p & -1.0446 & 0.0395 \\
hsa-miR-20a-5p & -1.0020 & 0.0097 \\
hsa-miR-3156-5p & 1.1413 & 0.0041 \\
hsa-miR-4660 & 1.1456 & 0.0036 \\
hsa-miR-4725-3p & 1.1457 & 0.0498 \\
hsa-miR-4271 & 1.1810 & 0.0134 \\
hsa-miR-1273e & 1.1995 & 0.0297 \\
hsa-miR-1273f & 1.2424 & 0.0023 \\
hsa-miR-551b-5p & 1.2642 & 0.0030 \\
hsa-miR-6514-5p & 1.3973 & 0.0011 \\
hsa-miR-3691-5p & 1.5138 & 0.0033 \\
hsa-miR-584-5p & 1.5218 & 0.0041 \\
hsa-miR-4430 & 2.3862 & 0.0071 \\
\hline
\end{tabular}

miR, microRNA.

separated from those of the unstimulated controls by hierarchical cluster analysis (Fig. 1).

Validation of miRNA by RT-qPCR. RT-qPCR was used to validate the results of two miRNAs that were identified through the microarray analysis. The results demonstrated that the expression levels of miR-363-3p and miR-551b-5p were significantly increased in ECs with mechanical stretch stimulation, compared with the control ECs (Fig. 2). This result supported the miRNA expression signatures identified in a previous experiment (Zheng et al, unpublished data). However, no novel miRNAs were identified.

$G O$ analysis. Using GO analysis of biological processes, the differentially expressed miRNAs were classified into different functional categories. As presented in Fig. 3, these miRNAs were identified to be involved in various vessel-associated processes, including positive regulation of nuclear factor- $\kappa \mathrm{B}$ transcription, and regulation of transcription factor activity, mitogen-activated protein kinase (MAPK) activity and blood pressure.

miRNA-associated regulatory networks. KEGG pathway analysis was conducted in order to determine the pathways associated with the differentially expressed miRNAs. It was observed that the miRNAs were involved in various pathways and proteins associated with vessel diseases, including the p53 signaling pathway, apoptosis, expression of cell adhesion molecules and chemokine signaling pathways (Fig. 4). As

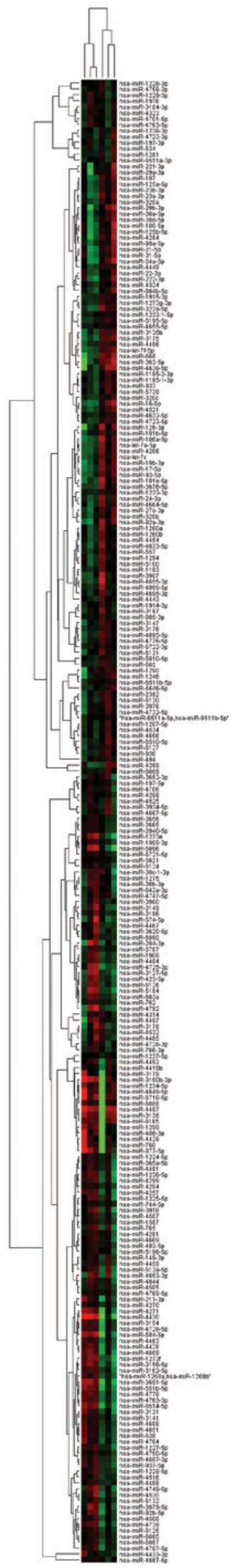

Figure 1. MicroRNA profiling in human umbilical vein endothelial cells with and without mechanical stretch stimulation. Red boxes represent upregulated genes, green boxes represent downregulated genes. 

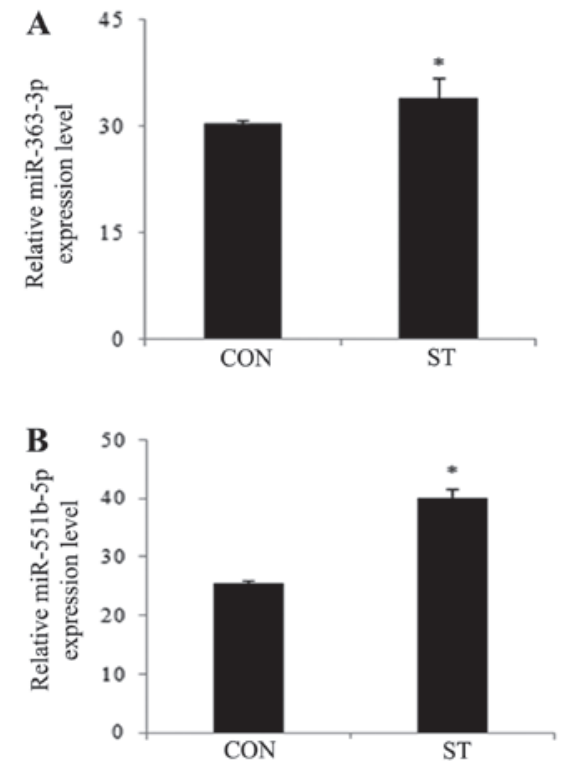

Figure 2. Validation of the microRNA expression levels by reverse transcription-quantitative polymerase chain reaction. (A) miR-363-3p, (B) miR-551b-5p. miR, microRNA; CON, normal endothelial cells; ST, endothelial cells with mechanical stretch stimulation. ${ }^{*} \mathrm{P}<0.05$ vs. control.

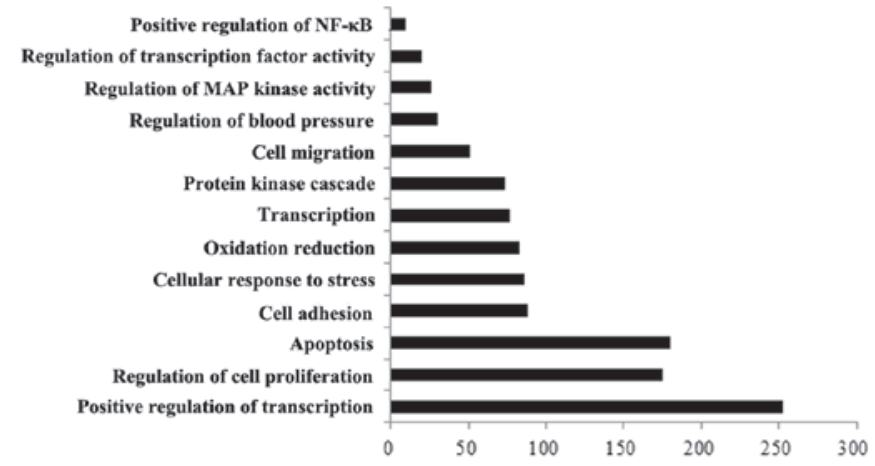

Figure 3. Selected GO functional categories of microRNAs differentially expressed by endothelial cells with mechanical stretch stimulation. The $\mathrm{y}$-axis is the GO category and the x-axis represents the number of genes in each category. GO, Gene Ontology; NF-кB; nuclear factor-кB; MAP, mitogen-activated protein.

demonstrated in Fig. 5, the differentially expressed miRNAs had a variety of potential targets.

\section{Discussion}

VGF is a serious complication that requires investigation in order to improve prognosis in cardiac surgery. Previous studies have demonstrated that subsequent to CABG surgery, VGF occurred in $25 \%$ of patients within 1 year and $50 \%$ of patients within 5 years (12). Compared with those undergoing their first CABG surgery, the mortality risk has been reported to increase 13 -fold in patients undergoing repeat CABG surgery due to VGF (13). Furthermore, diagnosing and treating VGF at early stages greatly improves patient prognosis (14).

miRNAs regulate gene expression by acting on the 3'-untranslated regions of target genes, and serve a central role in various physiological and pathological processes. Compared

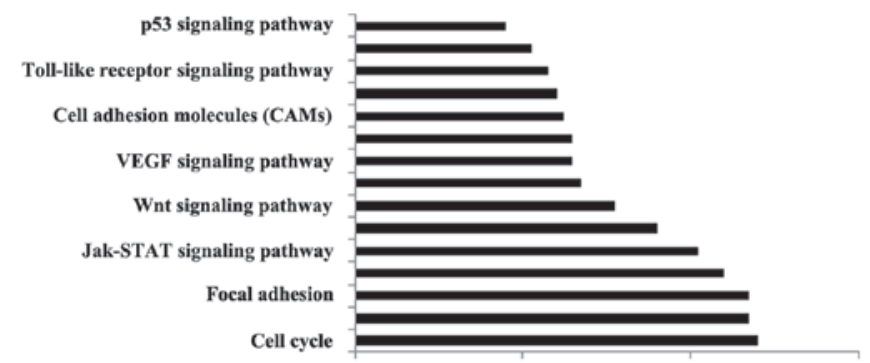

Figure 4. Pathway analysis based on microRNA-targeted genes. The y-axis is the pathway category and the $\mathrm{x}$-axis represents the number ofgenes in the pathway. VEGF, vascular endothelial growth factor; STAT, signal transducer and activator of transcription.
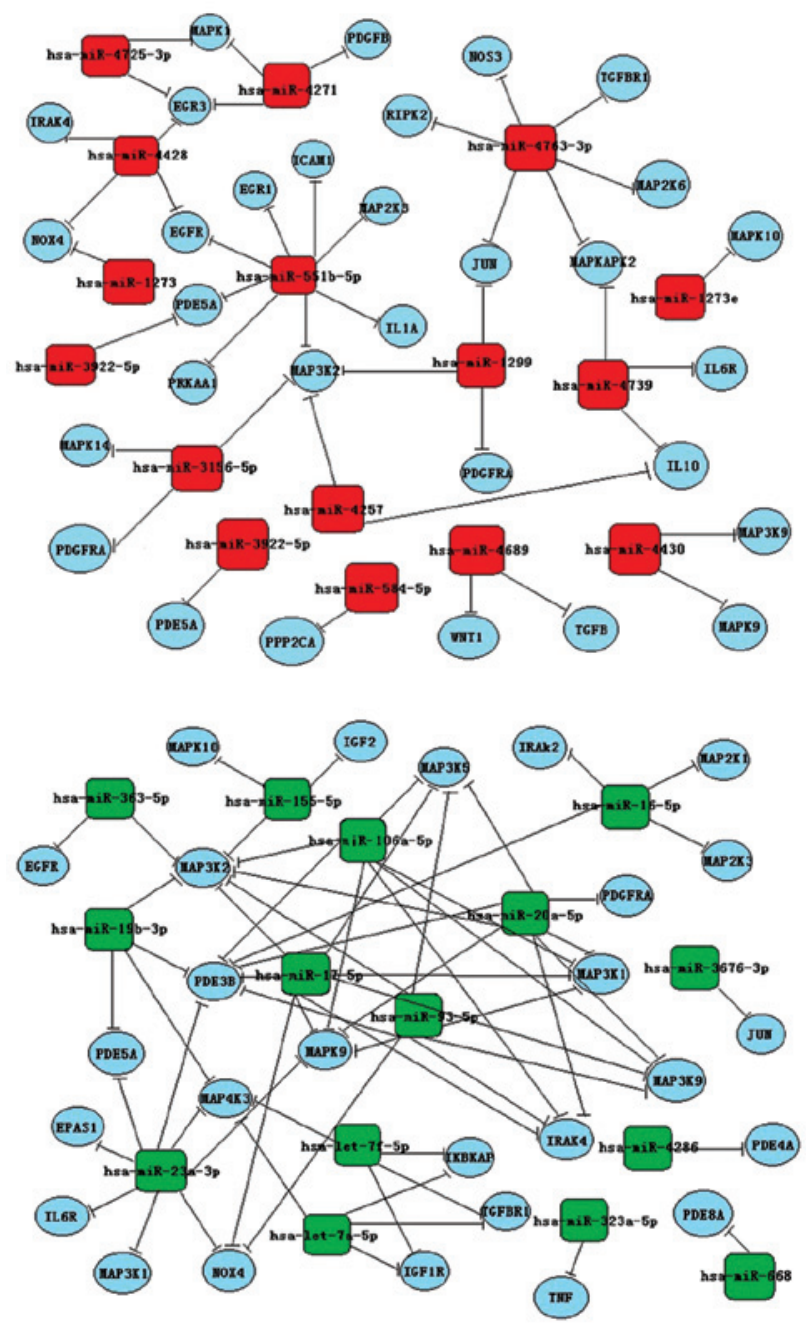

Figure 5. miRNA-mRNA network. Red boxes indicate the overexpression of miRNA, green boxes indicate downregulation of miRNA, and blue boxes represent potential target genes of the miRNAs. Edges represent the inhibitory effect of miRNA on mRNA. miRNA, microRNA.

with normal controls, differentially expressed miRNAs may be used as diagnostic biomarkers and therapeutic targets. For the purpose of elucidating the mechanism of endothelial dysfunction, the current study focused on an miRNA signature present in ECs stimulated by mechanical stretch. In total, 38 differentially expressed miRNAs were identified in these cells, including 20 that were upregulated and 18 that were 
downregulated. In order to ensure the quality of the array, certain miRNAs were validated by RT-qPCR. GO and KEGG pathway analysis was undertaken in order to predict the target genes and functional pathways of the differentially expressed miRNAs. GO analysis revealed that the target genes of these miRNAs were involved in pathological processes associated with VGF, including cell adhesion, cellular responses to stress and regulation of transcription. Of these genes, the most notable was EGR1. EGR1 is a gene that is expressed acutely at early VGF stages, with no sustained expression. Various previous studies have suggested that EGR1 is a critical transcription factor involved in the regulation of intracellular signaling, cell growth, differentiation and wound healing (15-17). In a previous study, it was observed that EGR1 is involved in VGF via regulating the promotion of EC proliferation, in addition to the expression of inflammatory factors following mechanical stretch stimulation (3). Additional studies have demonstrated that EGR1 expression may upregulate monocyte chemoattractant protein-1 expression, promote vascular inflammation and induce thrombosis and atherosclerosis (18). Additionally, EGR1 has been indicated to be capable of stimulating vascular smooth muscle cell (VSMC) growth, migration and apoptosis, and inducing VGF via the IGF-1 pathway (19).

KEGG analysis in the current study demonstrated that the transforming growth factor- $\beta$ (TGF- $\beta$ ) and MAPK signaling pathways were involved in VGF. In the early stages of vascular injury, TGF- $\beta$ is released by inflammatory cells and platelets, which attracts inflammatory factors and monocytes that secrete fibroblast growth factor, tumor necrosis factor (TNF) and interleukins. In turn, this promotes VSMC proliferation and migration and increases extracellular matrix production. In addition, TGF- $\beta$ may promote collagen type I and III composition, and induce intimal hyperplasia and vascular wall fibrosis (20). The MAPK signaling pathway is strongly associated with autogenous vein grafts and vein arterializations $(21,22)$. The MAPK family includes Jun N-terminal kinase, p38 and extracellular signal-regulated kinase, and these molecules are involved in cell growth, differentiation and apoptotic processes (22-24). Cornelissen et al (25) reported that mechanical stretch is an important factor for inducing $\mathrm{p} 38$-MAPK activation in pressure distension of human saphenous veins, while also identifying that p38-MAPK is associated with apoptosis. TNF- $\alpha$ stimulates TNF receptor 1 , activates MAPK expression and induces VSMC migration (26). Due to the fact that VSMC migration and apoptosis are important processes in VGF, Ge et al (27) were able to successfully use an MAPK inhibitor to limit intimal hyperplasia.

In the present study, 38 differentially expressed miRNAs were identified, a number of which had been previously reported to be associated with VGF. Zhi et al (28) identified a reduction of miR-106a-5p expression in astrocytoma, and its target gene Fas-activated serine/threonine kinase was upregulated, leading to poor survival outcomes in patients with astrocytoma. Costa et al (29) reported that bone marrow endothelial cells express miR-363-5p. It was observed that the miRNA was able to directly modulate the expression of the angiocrine factors tissue inhibitor of metalloproteinases- 1 and thrombospondin 3 at the post-transcriptional level. miR-363-5p downregulation additionally was observed to result in a significant reduction in EC tube formation on Matrigel, while increasing the adhesion of hematopoietic precursor cells to ECs (29).

VGF is a complex disease caused by multiple genes, factors and signaling pathways. An miRNA signature may provide a basis for investigating the mechanism of this disease, in addition to aiding in the identification of novel biomarkers or targets for diagnosis and therapy of early-stage VGF. In the current study, an miRNA signature was identified in ECs stimulated by mechanical stretch. The results of the present study suggest that miRNAs are vital in the process of endothelial dysfunction and are involved in the pathogenesis of VGF. Thus, using miRNAs as biomarkers and therapeutic targets has the potential to improve early diagnosis and prognosis of patients with VGF.

\section{Acknowledgements}

The current study was supported by the National Natural Science Foundation of China (grant no. 81070202).

\section{References}

1. Deb S, Wijeysundera HC, Ko DT, Tsubota H, Hill S and Fremes SE: Coronary artery bypass graft surgery vs percutaneous interventions in coronary revascularization: a systematic review. JAMA 310: 2086-2095, 2013.

2. Li FD, Sexton KW, Hocking KM, et al: Intimal thickness associated with endothelial dysfunction in human vein grafts. J Surg Res 180: e55-e62, 2013.

3. Zhang K, Cao J, Dong R and Du J: Early growth response protein 1 promotes restenosis by upregulating intercellular adhesion molecule-1 in vein graft. Oxid Med Cell Longev 2013: 432409, 2013.

4. Okada M, Matsumori A, Ono K, et al: Cyclic stretch upregulates production of interleukin- 8 and monocyte chemotactic and activating factor/monocyte chemoattractant protein-1 in human endothelial cells. Arterioscler Thromb Vasc Biol 18: 894-901, 1998.

5. Wu MH, Kouchi Y, Onuki Y, et al: Effect of differential shear stress on platelet aggregation, surface thrombosis, and endothelialization of bilateral carotid-femoral grafts in the dog. J Vasc Surg 22: 382-392, 1995.

6. Jinek $\mathrm{M}$ and Doudna JA: A three-dimensional view of the molecular machinery of RNA interference. Nature 457: 405-412, 2009.

7. Small EM and Olson EN: Pervasive roles of microRNAs in cardiovascular biology. Nature 469: 336-342, 2011.

8. Moazed D: Small RNAs in transcriptional gene silencing and genome defence. Nature 457: 413-420, 2009.

9. Lanford RE, Hildebrandt-Eriksen ES, Petri A, et al: Therapeutic silencing of microRNA-122 in primates with chronic hepatitis C virus infection. Science 327: 198-201, 2010.

10. McDonald RA, White KM, Wu J, et al: miRNA-21 is dysregulated in response to vein grafting in multiple models and genetic ablation in mice attenuates neointima formation. Eur Heart J 34: 1636-1643, 2013.

11. Stellos K and Dimmeler S: Vascular microRNAs: from disease mechanisms to therapeutic targets. Circ Res 114: 3-4, 2014.

12. Parang $P$ and Arora R: Coronary vein graft disease: pathogenesis and prevention. Can J Cardiol 25: e57-e62, 2009.

13. Harskamp RE, Lopes RD, Baisden CE, de Winter RJ and Alexander JH: Saphenous vein graft failure after coronary artery bypass surgery: pathophysiology, management and future directions. Ann Surg 257: 824-833, 2013.

14. Lopes RD, Mehta RH, Hafley GE, et al: Relationship between vein graft failure and subsequent clinical outcomes after coronary artery bypass surgery. Circulation 125: 749-756, 2012.

15. Rekhviashvili A and Abashidze R: The relationship between endothelial dysfunction and 24-hour blood pressure rhythm in patients with arterial hypertension. Georgian Med News 155: 13-17, 2008. 
16. Harja E, Bucciarelli LG, Lu Y, Stern DM, Zou YS, Schmidt AM and Yan SF: Early growth response-1 promotes atherogenesis: mice deficient in early growth response- 1 and apolipoprotein $\mathrm{E}$ display decreased atherosclerosis and vascular inflammation. Circ Res 94: 333-339, 2004.

17. Fujita T, Asai T, Andrassy M, et al: PKCbeta regulates ischemia/reperfusion injury in the lung. J Clin Invest 113: $1615-1623,2004$.

18. Maekawa T, Takahashi N, Honda T, et al: Porphyromonas gingivalis antigens and interleukin- 6 stimulate the production of monocyte chemoattractant protein-1 via the upregulation of early growth response- 1 transcription in human coronary artery endothelial cells. J Vasc Res 47: 346-354, 2010.

19. Wu X,Cheng J,Li P, Yang M, Qiu S,Liu Pand Du J: Mechano-sensitive transcriptional factor Egr-1 regulates insulin-like growth factor-1 receptor expression and contributes to neointima formation in vein grafts. Arterioscler Thromb Vasc Biol 30: 471-476, 2010.

20. Marchesi C, Paradis P and Schiffrin EL: Role of the renin-angiotensin system in vascular inflammation. Trends Pharmacol Sci 29: 367-374, 2008.

21. Pintucci G, Saunders PC, Gulkarov I, et al: Anti-proliferative and anti-inflammatory effects of topical MAPK inhibition in arterialized vein grafts. FASEB J 20: 398-400, 2006

22. Saunders PC, Pintucci G, Bizekis CS, et al: Vein graft arterialization causes differential activation of mitogen-activated protein kinases. J Thorac Cardiovasc Surg 127: 1276-1284, 2004.
23. Pearson G, Robinson F, Beers Gibson T, Xu BE, Karandikar M, Berman K and Cobb MH: Mitogen-activated protein (MAP) kinase pathways: regulation and physiological functions. Endocr Rev 22: 153-183, 2001

24. Wang XZ and Ron D: Stress-induced phosphorylation and activation of the transcription factor CHOP (GADD153) by p38 MAP Kinase. Science 272: 1347-1349, 1996.

25. Cornelissen J, Armstrong J and Holt CM: Mechanical stretch induces phosphorylation of p38-MAPK and apoptosis in human saphenous vein. Arterioscler Thromb Vasc Biol 24: 451-456, 2004.

26. Goetze S, Xi XP, Kawano Y, Kawano H, Fleck E, Hsueh WA and Law RE: TNF-alpha-induced migration of vascular smooth muscle cells is MAPK dependent. Hypertension 33: 183-189, 1999.

27. Ge JJ, Zhao ZW, Zhou ZC, Wu S, Zhang R, Pan FM and Abendroth DK: p38 MAPK inhibitor, CBS3830 limits vascular remodelling in arterialised vein grafts. Heart Lung Circ 22: 751-758, 2013.

28. Zhi F, Zhou G, Shao N, et al: miR-106a-5p inhibits the proliferation and migration of astrocytoma cells and promotes apoptosis by targeting FASTK. PLoS One 8: e72390, 2013.

29. Costa A, Afonso J, Osório C, et al: miR-363-5p regulates endothelial cell properties and their communication with hematopoietic precursor cells. J Hematol Oncol 6: 87, 2013. 